\title{
On F-Polynomial, Multiple and Hyper F-Index of Some Molecular Graphs
}

\author{
Sirous Ghobadi ${ }^{1, \mathrm{a}}$, Mobina Ghorbaninejad ${ }^{1, \mathrm{~b}}$,
}

${ }^{1}$ Department of Mathematics, Qaemshahr Branch, Islamic Azad University, Qaemshahr, Iran

aghobadimath46@gmail.com, bghorbani325@gmail.com

Keywords: Topological index, F-polynomial, multiple F-index, Hyper F-index.

\begin{abstract}
A graph can be recognized by a numeric number, a polynomial, a sequence of numbers or a matrix which represent the whole graph, and these representations are aimed to be uniquely defined for that graph. Topological index is a numeric quantity with a graph which characterizes the topology of the graph and is invariant under graph automorphism. In this paper, we compute F-polynomial, Multiple F-index and Hyper F-index for some special graphs.
\end{abstract}

\section{Introduction}

A graph $G=(V, E)$ with vertex set $V$ and edge set $E$ is connected, if there exists a connection between any pair of vertices in $G$. For a graph $G$, the degree of a vertex $v$ is the number of edges incident with $v$ and denoted by $d_{G}(v)$.

Topological indices are numbers associated with molecular graphs for the purpose of allowing quantitative structure-activity/property relationships [6]. Topological indices correlate certain physicochemical properties like boiling point, stability, strain energy etc of chemical compounds.

The idea of topological index appears from work done by Harold Wiener (see [9]) in 1947 although he was working on boiling point of Paraffin. He called this index as Wiener index and then theory of topological index started. A pair of molecular descriptors known as the first Zagreb index $M_{1}(G)$ and the second Zagreb index $M_{2}(G)$, first appeared in the topological formula for the total $\pi$ - electron energy of conjugated molecules that has been derived in 1972 by I. Gutman and N. Trinajstic(see [5]). The first Zagreb index $M_{1}(G)$ and the second Zagreb index $M_{2}(G)$ of a molecular graph $G$ are respectively defined as

$$
M_{1}(G)=\sum_{u \in V(G)} d_{G}^{2}(u)=\sum_{u v \in E(G)}\left[d_{G}(u)+d_{G}(v)\right]
$$

and

$$
M_{2}(G)=\sum_{u v \in E(G)} d_{G}(u) d_{G}(v) .
$$

In the same paper, another topological index, defined as the sum of cubed degrees of the vertices of a graph, was also shown to relate $\pi$-electron energy. However, this index was never studied in detail then. In 2015, Furtula and Gutman [2] have restudied this index to establish some basic properties and have also demonstrated that the predictive ability of this index is similar to that of the first Zagreb index with respect to entropy and acetic factors of the molecules and both first Zagreb index and this index yield correlation coefficients greater than 0.95 . They named this index the "Forgotten topological index" or "F-index". In symbolic notation, the $F$-index is given as follows:

$$
F(G)=\sum_{u \in V(G)} d_{G}^{3}(u)=\sum_{u v \in E(G)}\left[d_{G}^{2}(u)+d_{G}^{2}(v)\right] .
$$

Similar to the first Zagreb polynomial [4], first multiple Zagreb index [3] and hyper Zagreb index [8], the $F$-polynomial, multiple F-index and hyper $F$-index of a graph $G$ respectively are defined as $F(G, x)=\sum_{u v \in E(G)} x^{\left[d_{G}^{2}(u)+d_{G}^{2}(v)\right]}$ 
$P F(G)=\prod_{u v \in E(G)}\left[d_{G}^{2}(u)+d_{G}^{2}(v)\right]$
$H F(G)=\sum_{u v \in E(G)}\left[d_{G}^{2}(u)+d_{G}^{2}(v)\right]^{2}$.

Siddiqui et al. in [7] presented the Zagreb indices and Zagreb polynomials of different nanostar dendrimers. In [1], N.De et al. computed F-index and F-polynomial of six infinite classes of dendrimer nanostars, namely: $N S_{1}[n], N S_{2}[n], N S_{3}[n], N S_{4}[n], N S_{5}[n]$ and $D_{n}$. In this paper, we obtain, F-polynomial, multiple and hyper F-index for some molecular graphs namely Dandelion graph, Friendship graph, Comet graph, $t$-fold bristled graph of $P_{n}$ and $C_{n}$, Fence and Closed fence graph, Tadpole graph, Ladder graph and Wheel.

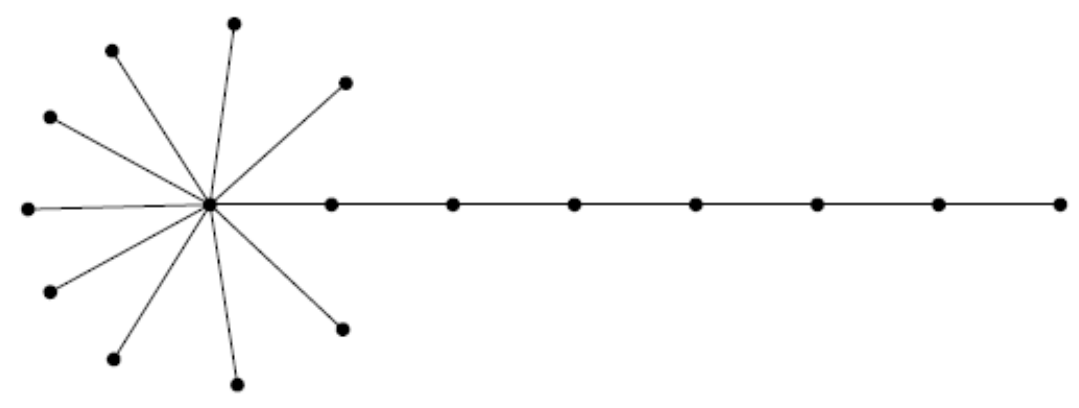

Fig. 1: Dandelion graph $D(17,8)$

\section{Main Results}

Let $D(n, l)$ be a Dandelion graph with $n$ vertices, consisted of a copy of the star $S_{n-l}$ and a copy of a path $P_{l}$ with vertices $p_{0}, p_{1}, \ldots p_{l-1}$, where $p_{0}$ is identified with a star center. (See Fig. 1)

Theorem 1. Let $D(n, l)$ be a Dandelion graph. Then

$$
\begin{gathered}
F(D(n, l), x)=x^{5}+(n-l) x^{(n-l+1)^{2}+1}+x^{(n-l+1)^{2}+4}+(l-3) x^{8} \\
P F(D(n, l))=5 \times\left[1+(n-l+1)^{2}\right]^{(n-l)} \times\left[4+(n-l+1)^{2}\right] \times 8^{(l-3)} \\
H F(D(n, l))=25+(n-l)\left[1+(n-l+1)^{2}\right]^{2}+\left[4+(n-l+1)^{2}\right]^{2}+64(l-3) .
\end{gathered}
$$

Proof. Let $G$ be a Dandelion graph $D(n, l)$. The edge set of $G$ is divided into four edge partition based on the degrees of end vertices. We have

$$
E(G)=\bigcup_{i=1}^{4} E_{i}(G)
$$

where

$$
\begin{gathered}
E_{1}(G)=\left\{e=u v \in E(G): d_{G}(u)=1 \text { and } d_{G}(v)=2\right\} \\
E_{2}(G)=\left\{e=u v \in E(G): d_{G}(u)=1 \text { and } d_{G}(v)=n-l+1\right\} \\
E_{3}(G)=\left\{e=u v \in E(G): d_{G}(u)=n-l+1 \text { and } d_{G}(v)=2\right\} \\
E_{4}(G)=\left\{e=u v \in E(G): d_{G}(u)=2 \text { and } d_{G}(v)=2\right\} .
\end{gathered}
$$


Hence, $\left|E_{1}(G)\right|=1,\left|E_{2}(G)\right|=n-l,\left|E_{3}(G)\right|=1$ and $\left|E_{4}(G)\right|=l-3$. Now using Eqs.(1)-(3), we have

$$
\begin{aligned}
& F(G, x)=\sum_{u v \in E(G)} x^{\left[d_{G}^{2}(u)+d_{G}^{2}(v)\right]} \\
& =\sum_{u v \in E_{1}(G)} x^{\left[d_{G}^{2}(u)+d_{G}^{2}(v)\right]}+\sum_{u v \in E_{2}(G)} x^{\left[d_{G}^{2}(u)+d_{G}^{2}(v)\right]} \\
& +\sum_{u v \in E_{3}(G)} x^{\left[d_{G}^{2}(u)+d_{G}^{2}(v)\right]}+\sum_{u v \in E_{4}(G)} x^{\left[d_{G}^{2}(u)+d_{G}^{2}(v)\right]} \\
& =\sum_{u v \in E_{1}(G)} x^{5}+\sum_{u v \in E_{2}(G)} x^{(n-l+1)^{2}+1}+\sum_{u v \in E_{3}(G)} x^{(n-l+1)^{2}+4}+\sum_{u v \in E_{4}(G)} x^{8} \\
& =\left|E_{1}(G)\right| x^{5}+\left|E_{2}(G)\right| x^{(n-l+1)^{2}+1}+\left|E_{3}(G)\right| x^{(n-l+1)^{2}+4}+\left|E_{4}(G)\right| x^{8} \\
& =x^{5}+(n-l) x^{(n-l+1)^{2}+1}+x^{(n-l+1)^{2}+4}+(l-3) x^{8} \\
& P F(G)=\prod_{u v \in E(G)}\left[d_{G}^{2}(u)+d_{G}^{2}(v)\right] \\
& =\prod_{u v \in E_{1}(G)}\left[d_{G}^{2}(u)+d_{G}^{2}(v)\right] \times \prod_{u v \in E_{2}(G)}\left[d_{G}^{2}(u)+d_{G}^{2}(v)\right] \\
& \times \prod_{u v \in E_{3}(G)}\left[d_{G}^{2}(u)+d_{G}^{2}(v)\right] \times \prod_{u v \in E_{4}(G)}\left[d_{G}^{2}(u)+d_{G}^{2}(v)\right] \\
& =5^{\left|E_{1}(G)\right|} \times\left[(n-l+1)^{2}+1\right]^{\left|E_{2}(G)\right|} \times\left[(n-l+1)^{2}+4\right]^{\left|E_{3}(G)\right|} \times 8^{\left|E_{4}(G)\right|} \\
& =5 \times\left[(n-l+1)^{2}+1\right]^{(n-l)} \times\left[(n-l+1)^{2}+4\right] \times 8^{(l-3)} \\
& H F(G)=\sum_{u v \in E(G)}\left[d_{G}^{2}(u)+d_{G}^{2}(v)\right]^{2} \\
& =\sum_{u v \in E_{1}(G)}\left[d_{G}^{2}(u)+d_{G}^{2}(v)\right]^{2}+\sum_{u v \in E_{2}(G)}\left[d_{G}^{2}(u)+d_{G}^{2}(v)\right]^{2} \\
& +\sum_{u v \in E_{3}(G)}\left[d_{G}^{2}(u)+d_{G}^{2}(v)\right]^{2}+\sum_{u v \in E_{4}(G)}\left[d_{G}^{2}(u)+d_{G}^{2}(v)\right]^{2} \\
& =25\left|E_{1}(G)\right|+\left[(n-l+1)^{2}+1\right]^{2}\left|E_{2}(G)\right|+\left[(n-l+1)^{2}+4\right]^{2}\left|E_{3}(G)\right|+64\left|E_{4}(G)\right| \\
& =25+(n-l)\left[(n-l+1)^{2}+1\right]^{2}+\left[(n-l+1)^{2}+4\right]^{2}+64(l-3) \text {. }
\end{aligned}
$$

A friendship graph (or Dutch windmill graph) $F_{m}$ is a graph with $2 m+1$ vertices and $3 m$ edges constructed by joining $m$ copies of the cycle graph $C_{3}$ with a common vertex.(See Fig. 2)

Theorem 2. Let $F_{m}$ be a friendship graph. Then

$$
\begin{gathered}
F\left(F_{m}, x\right)=2 m x^{4 m^{2}+4}+m x^{8} \\
P F\left(F_{m}\right)=\left(4+4 m^{2}\right)^{2 m} \times 8^{m} \\
H F\left(F_{m}\right)=2 m\left(4+4 m^{2}\right)^{2}+64 m .
\end{gathered}
$$




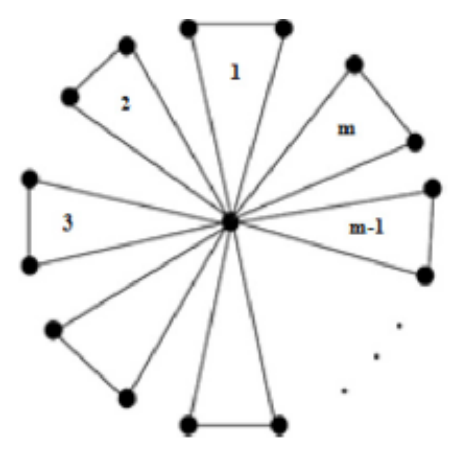

Fig. 2: Friendship graph $F_{m}$

Proof. Let $G$ be a friendship graph $F_{m}$. The edge set of $G$ is divided into two edge partition based on degrees of end vertices as follow:

$$
\begin{gathered}
E_{1}(G)=\left\{e=u v \in E(G): d_{G}(u)=2 m \text { and } d_{G}(v)=2\right\} \\
E_{2}(G)=\left\{e=u v \in E(G): d_{G}(u)=2 \text { and } d_{G}(v)=2\right\} .
\end{gathered}
$$

Hence, $\left|E_{1}(G)\right|=2 m$ and $\left|E_{2}(G)\right|=m$. Now using Eqs.(1)-(3) we have

$$
\begin{aligned}
F(G, x) & =\sum_{u v \in E(G)} x^{\left[d_{G}^{2}(u)+d_{G}^{2}(v)\right]} \\
& =\sum_{u v \in E_{1}(G)} x^{\left[d_{G}^{2}(u)+d_{G}^{2}(v)\right]}+\sum_{u v \in E_{2}(G)} x^{\left[d_{G}^{2}(u)+d_{G}^{2}(v)\right]} \\
& =\sum_{u v \in E_{1}(G)} x^{4 m^{2}+4}+\sum_{u v \in E_{2}(G)} x^{8} \\
& =\left|E_{1}(G)\right| x^{4 m^{2}+4}+\left|E_{2}(G)\right| x^{8} \\
& =2 m x^{4 m^{2}+4}+m x^{8} \\
P F(G) & =\prod_{u v \in E(G)}\left[d_{G}^{2}(u)+d_{G}^{2}(v)\right] \\
& =\prod_{u v \in E_{1}(G)}\left[d_{G}^{2}(u)+d_{G}^{2}(v)\right] \times \prod_{u v \in E_{2}(G)}\left[d_{G}^{2}(u)+d_{G}^{2}(v)\right] \\
& =\left(4+4 m^{2}\right)^{\left|E_{1}(G)\right|} \times 8^{\left|E_{2}(G)\right|} \\
& =\left(4+4 m^{2}\right)^{2 m} \times 8^{m} \\
& =\sum_{u v \in E(G)}\left[d_{G}^{2}(u)+d_{G}^{2}(v)\right]^{2} \\
& =\sum_{u v \in E_{1}(G)}\left[d_{G}^{2}(u)+d_{G}^{2}(v)\right]^{2}+\sum_{u v \in E_{2}(G)}\left[d_{G}^{2}(u)+d_{G}^{2}(v)\right]^{2} \\
H F(G) & 2 m\left(4+4 m^{2}\right)^{2}\left|E_{1}(G)\right|+64\left|E_{2}(G)\right| \\
& =64 m .
\end{aligned}
$$

For a positive integer $l \leq n$ let $C(n, l)$ be the comet graph with $n$ vertices, consisted of a copy of the complete graph $K_{n-l+1}$ and a copy of the path $P_{l}$, with vertices $p_{0}, p_{1}, \ldots, p_{l-1}$, where $p_{0}$ is identified with a vertex from $K_{n-l+1}$.(See Fig. 3) 


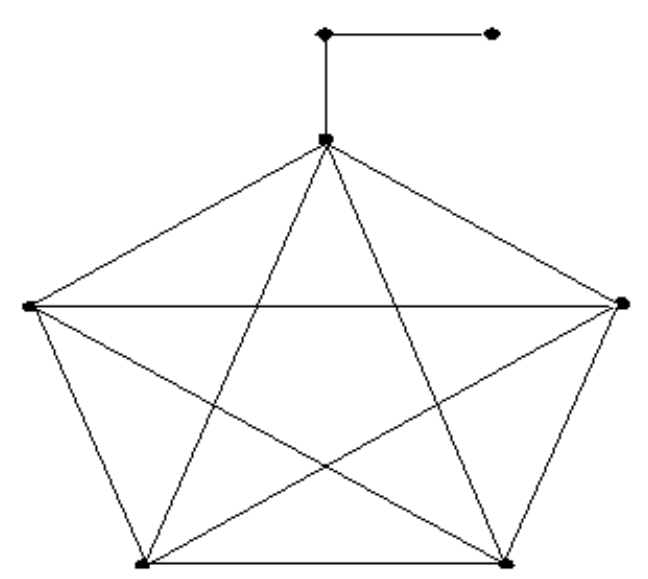

Fig. 3: Comet graph $C(7,3)$

Theorem 3. Let $C(n, l)$ be a comet graph. Then

$F(C(n, l), x)=x^{5}+(l-3) x^{8}+(n-l) x^{(n-l+1)^{2}+(n-l)^{2}}+\frac{(n-l)(n-l-1)}{2} x^{2(n-l)^{2}}+x^{(n-l+1)^{2}+4}$

$P F(C(n, l))=5 \times 8^{(l-3)} \times\left[(n-l+1)^{2}+(n-l)^{2}\right]^{(n-l)} \times\left[2(n-l)^{2}\right]^{\frac{(n-l)(n-l-1)}{2}} \times\left[(n-l+1)^{2}+4\right]$ $H F(C(n, l))=25+64(l-3)+(n-l)\left[(n-l+1)^{2}+(n-l)^{2}\right]^{2}+2(n-l)^{4}[(n-l)(n-l-1)]+\left[4+(n-l+1)^{2}\right]^{2}$.

Proof. Let $G$ be a Comet graph $C(n, l)$. The edge set of $G$ is divided into five partition based on degrees of end vertices. We have

$$
\begin{gathered}
E_{1}(G)=\left\{e=u v \in E(G): d_{G}(u)=1 \text { and } d_{G}(v)=2\right\} \\
E_{2}(G)=\left\{e=u v \in E(G): d_{G}(u)=2 \text { and } d_{G}(v)=2\right\} \\
E_{3}(G)=\left\{e=u v \in E(G): d_{G}(u)=n-l+1 \text { and } d_{G}(v)=n-l\right\} \\
E_{4}(G)=\left\{e=u v \in E(G): d_{G}(u)=n-l \text { and } d_{G}(v)=n-l\right\} \\
E_{5}(G)=\left\{e=u v \in E(G): d_{G}(u)=2 \text { and } d_{G}(v)=n-l+1\right\} .
\end{gathered}
$$

Hence, $\left|E_{1}(G)\right|=1,\left|E_{2}(G)\right|=l-3,\left|E_{3}(G)\right|=n-l,\left|E_{4}(G)\right|=\frac{(n-l)(n-l-1)}{2}$ and $\left|E_{5}(G)\right|=1$. Similar to the above theorems and using Eqs.(1)-(3) we have

$$
\begin{aligned}
F(G, x) & =\sum_{u v \in E_{1}(G)} x^{\left[d_{G}^{2}(u)+d_{G}^{2}(v)\right]}+\ldots+\sum_{u v \in E_{5}(G)} x^{\left[d_{G}^{2}(u)+d_{G}^{2}(v)\right]} \\
& =x^{5}+(l-3) x^{8}+(n-l) x^{(n-l+1)^{2}+(n-l)^{2}}+\frac{(n-l)(n-l-1)}{2} x^{2(n-l)^{2}}+x^{(n-l+1)^{2}+4} \\
P F(G) & =\prod_{u v \in E_{1}(G)}\left[d_{G}^{2}(u)+d_{G}^{2}(v)\right] \times \ldots \times \prod_{u v \in E_{5}(G)}\left[d_{G}^{2}(u)+d_{G}^{2}(v)\right] \\
& =5 \times 8^{(l-3)} \times\left[(n-l+1)^{2}+(n-l)^{2}\right]^{(n-l)} \times\left[2(n-l)^{2}\right]^{\frac{(n-l)(n-l-1)}{2}} \times\left[(n-l+1)^{2}+4\right] \\
H F(G) & =\sum_{u v \in E_{1}(G)}\left[d_{G}^{2}(u)+d_{G}^{2}(v)\right]^{2}+\ldots+\sum_{u v \in E_{5}(G)}\left[d_{G}^{2}(u)+d_{G}^{2}(v)\right]^{2} \\
& =25+64(l-3)+(n-l)\left[(n-l+1)^{2}+(n-l)^{2}\right]^{2}+2(n-l)^{5}(n-l-1) \\
& +\left[4+(n-l+1)^{2}\right]^{2} .
\end{aligned}
$$



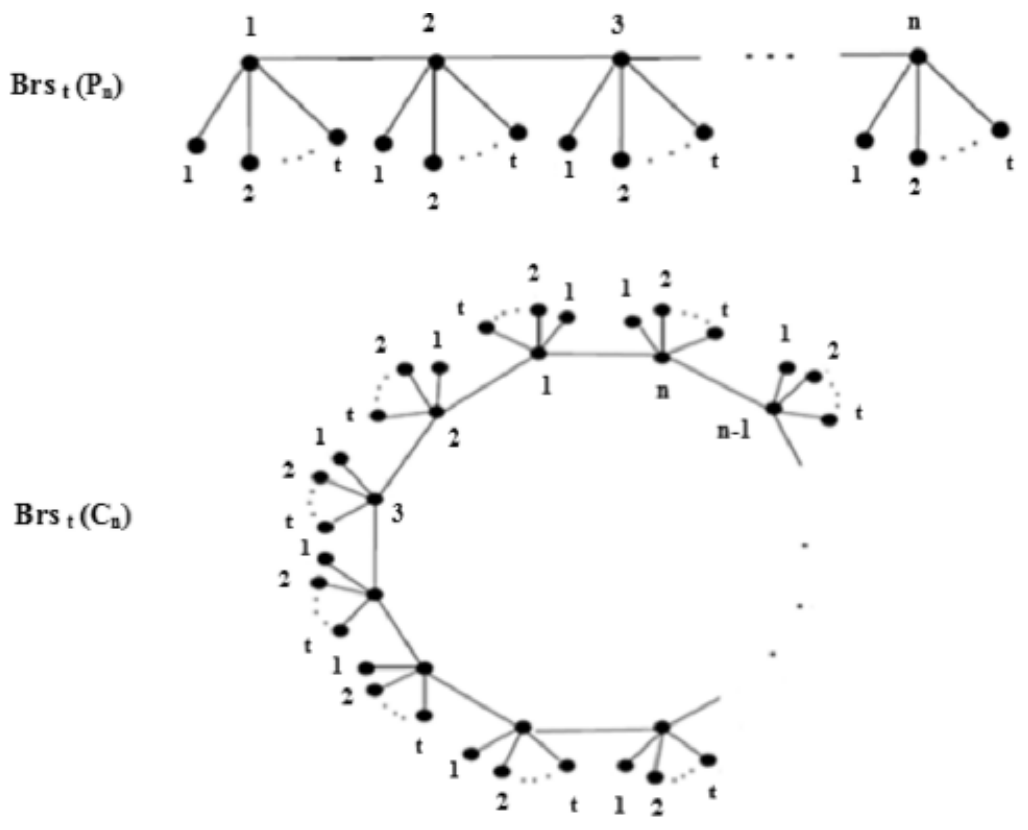

Fig. 4: t-fold bristled graph of $P_{n}$ and $C_{n}$

For a given graph $G$, its t-fold bristled graph $B r s_{t}(G)$ is obtained by attaching $t$ vertices of degree one to each vertex of $G$.

Theorem 4. Let $\operatorname{Brs}_{t}\left(P_{n}\right)$ be a t-fold bristled graph of $P_{n}$ (See Fig. 4). Then

$$
\begin{gathered}
F\left(\operatorname{Brs}_{t}\left(P_{n}\right)\right)=2 t x^{(t+1)^{2}+1}+t(n-2) x^{(t+2)^{2}+1}+2 x^{(t+1)^{2}+(t+2)^{2}}+(n-3) x^{2(t+2)^{2}} \\
P F\left(\operatorname{Brs}_{t}\left(P_{n}\right)\right)=\left[(t+1)^{2}+1\right]^{2 t} \times\left[(t+2)^{2}+1\right]^{t(n-2)} \times\left[(t+1)^{2}+(t+2)^{2}\right]^{2} \times\left[2(t+2)^{2}\right]^{n-3} \\
H F\left(\operatorname{Brs}_{t}\left(P_{n}\right)\right)=2 t\left[(t+1)^{2}+1\right]^{2}+t(n-2)\left[(t+2)^{2}+1\right]^{2}+2\left[(t+1)^{2}+(t+2)^{2}\right]^{2}+4(n-3)(t+2)^{4} .
\end{gathered}
$$

Proof. Let $G$ be a t-fold Bristled graph of $P_{n}$. The edge set of $G$ is divided into four partition based on degrees of end vertices. we have

$$
E_{1}(G)=\left\{e=u v \in E(G): d_{G}(u)=1 \text { and } d_{G}(v)=t+1\right\}
$$

$E_{2}(G)=\left\{e=u v \in E(G): d_{G}(u)=1\right.$ and $\left.d_{G}(v)=t+2\right\}$

$E_{3}(G)=\left\{e=u v \in E(G): d_{G}(u)=t+1\right.$ and $\left.d_{G}(v)=t+2\right\}$

$E_{4}(G)=\left\{e=u v \in E(G): d_{G}(u)=t+2\right.$ and $\left.d_{G}(v)=t+2\right\}$.

Hence, $\left|E_{1}(G)\right|=2 t,\left|E_{2}(G)\right|=t(n-2),\left|E_{3}(G)\right|=2$ and $\left|E_{4}(G)\right|=n-3$. Similar to the above theorems and using Eqs.(1)-(3) the proof is easy.

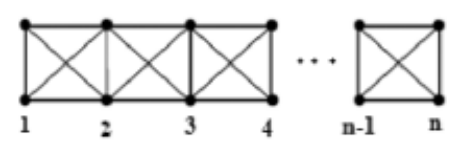

$\mathrm{P}_{\mathrm{u}}\left[\mathrm{P}_{2}\right]$

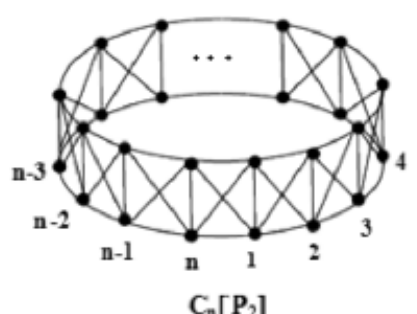

$\mathrm{C}_{\mathrm{u}}\left[\mathrm{P}_{2}\right]$

Fig. 5: Fence and Closed fence graphs

For the Fence graph $P_{n}\left[P_{2}\right]$ (See Fig. 5), we can divided the edge set into three edge partition based on degrees of end vertices. we have 


$$
\begin{aligned}
& E_{1}\left(P_{n}\left[P_{2}\right]\right)=\left\{e=u v \in E\left(P_{n}\left[P_{2}\right]\right): d_{G}(u)=3 \text { and } d_{G}(v)=3\right\} \\
& E_{2}\left(P_{n}\left[P_{2}\right]\right)=\left\{e=u v \in E\left(P_{n}\left[P_{2}\right]\right): d_{G}(u)=3 \text { and } d_{G}(v)=5\right\} \\
& E_{3}\left(P_{n}\left[P_{2}\right]\right)=\left\{e=u v \in E\left(P_{n}\left[P_{2}\right]\right): d_{G}(u)=5 \text { and } d_{G}(v)=5\right\} .
\end{aligned}
$$

Hence, $\left|E_{1}\left(P_{n}\left[P_{2}\right]\right)\right|=2,\left|E_{2}\left(P_{n}\left[P_{2}\right]\right)\right|=8$ and $\left|E_{3}\left(P_{n}\left[P_{2}\right]\right)\right|=5 n-14$. Using Eqs.(1)-(3), we have,

Theorem 5. Let $P_{n}\left[P_{2}\right]$ be a Fence graph with $2 n$ vertices. Then

$$
\begin{gathered}
F\left(P_{n}\left[P_{2}\right], x\right)=2 x^{18}+8 x^{34}+(5 n-14) x^{50} \\
P F\left(P_{n}\left[P_{2}\right]\right)=18^{2} \times 34^{8} \times 50^{5 n-14} \\
H F\left(P_{n}\left[P_{2}\right]\right)=9896+2500(5 n-14) .
\end{gathered}
$$

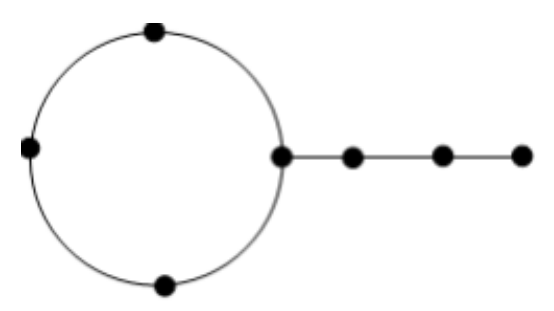

Fig. 6: Tadpole graph $T_{4,3}$

A Tadpole graph $T_{n, k}$ is a special type of graph consisting of a cycle graph on $n$ (at least 3 ) vertices and a path graph with $k$ vertices, connected with a bridge.(See Fig. 6) For the Tadpole graph $T_{n, k}$ we can divide the edge set into three partition based on degrees of end vertices as follows:

$E_{1}\left(T_{n, k}\right)=\left\{e=u v \in E\left(P_{n}\left[P_{2}\right]\right): d_{G}(u)=2\right.$ and $\left.d_{G}(v)=2\right\}$

$E_{2}\left(T_{n, k}\right)=\left\{e=u v \in E\left(P_{n}\left[P_{2}\right]\right): d_{G}(u)=3\right.$ and $\left.d_{G}(v)=2\right\}$

$E_{3}\left(T_{n, k}\right)=\left\{e=u v \in E\left(P_{n}\left[P_{2}\right]\right): d_{G}(u)=1\right.$ and $\left.d_{G}(v)=2\right\}$.

Hence, $\left|E_{1}\left(T_{n, k}\right)\right|=n+k-4,\left|E_{2}\left(T_{n, k}\right)\right|=3$ and $\left|E_{3}\left(T_{n, k}\right)\right|=1$. Using Eqs.(1)-(3) we have,

Theorem 6. Let $T_{n, k}$ be a Tadpole graph. Then

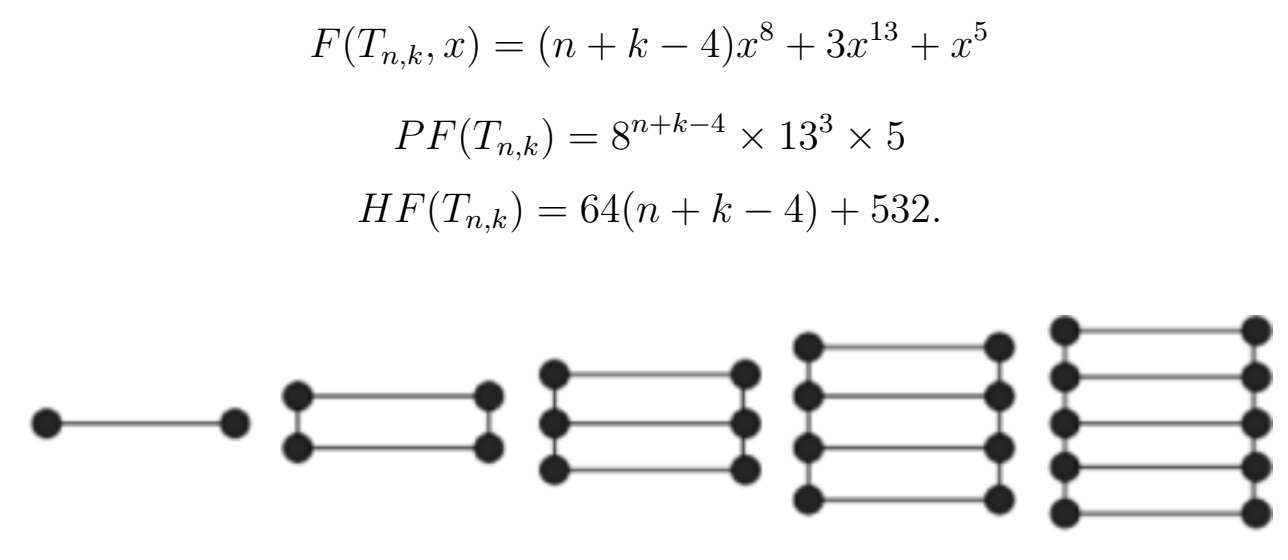

Fig. 7: Ladder graphs $L_{1}, L_{2}, L_{3}, L_{4}, L_{5}$

With reference to the above theorems the proofs of the next four results are easy. So we omit the proofs. 
Theorem 7. Let $L_{n}$ be a Ladder graph obtained as the Cartesian product of two path graphs, one of which has only one edge(See Fig. 7). Then

$$
\begin{gathered}
F\left(L_{n}, x\right)=2 x^{8}+4 x^{13}+(n-2) x^{18} \\
P F\left(L_{n}\right)=8^{2} \times 13^{4} \times 18^{n-2} \\
H F\left(L_{n}\right)=804+324(n-2) .
\end{gathered}
$$

Theorem 8. Let Brs $s_{t}\left[C_{n}\right]$ be a t-fold bristled graph of $C_{n}($ See Fig. 4). Then

$$
\begin{gathered}
F\left(B r s_{t}\left[C_{n}\right], x\right)=\operatorname{tn} x^{(t+2)^{2}+1}+n x^{2(t+2)^{2}} \\
\operatorname{PF}\left(B r s_{t}\left[C_{n}\right]\right)=\left[(t+2)^{2}+1\right]^{t n} \times\left[2(t+2)^{2}\right]^{n} \\
H F\left(B r s_{t}\left[C_{n}\right]\right)=\operatorname{tn}\left[(t+2)^{2}+1\right]^{2}+4 n(t+2)^{4} .
\end{gathered}
$$

Theorem 9. Let $C_{n}\left[P_{2}\right]$ be a Closed fence graph with $2 n$ vertices(See Fig. 5). Then

$$
\begin{gathered}
F\left(C_{n}\left[P_{2}\right], x\right)=(5 n-2) x^{50} \\
P F\left(C_{n}\left[P_{2}\right]\right)=50^{5 n-2} \\
H F\left(C_{n}\left[P_{2}\right]\right)=2500(5 n-2) .
\end{gathered}
$$

Theorem 10. Let $W_{n+1}$ be a Wheel graph. Then

$$
\begin{gathered}
F\left(W_{n+1}, x\right)=n x^{18}+n x^{n^{2}+9} \\
P F\left(W_{n+1}\right)=18^{n} \times\left[9+n^{2}\right]^{n} \\
H F\left(W_{n+1}\right)=324 n+n\left[9+n^{2}\right]^{2} .
\end{gathered}
$$

Having computed above indices, it is not difficult for computing other degree-based indices such as Randic index, Multiplicative Zagreb indices etc., for the graphs applied in this article. For instance, in the following two theorems we compute Randic polynomial, Multiple Randic index, and Hyper Randic index for the Fence graph and Tadpole graph. The proofs are easy, so we omit the proofs.

The Randic polynomial, Multiple Randic index, and Hyper Randic index of a graph $G$ respectively are defined as

$$
\begin{aligned}
& \chi(G, x)=\sum_{u v \in E(G)} x^{\frac{1}{\sqrt{d(u) d(v)}}} \\
& P \chi(G(x))=\sum_{u v \in E(G)} \frac{1}{\sqrt{d(u) d(v)}} \\
& H \chi(G(x))=\sum_{u v \in E(G)}\left(\frac{1}{\sqrt{d(u) d(v)}}\right)^{2} .
\end{aligned}
$$

Theorem 11. Let $P_{n}\left[P_{2}\right]$ be a Fence graph with $2 n$ vertices. Then

$$
\begin{gathered}
\chi\left(P_{n}\left[P_{2}\right], x\right)=2 x^{\frac{1}{3}}+8 x^{\frac{1}{\sqrt{15}}}+(5 n-14) x^{\frac{1}{5}} \\
P \chi\left(P_{n}\left[P_{2}\right]\right)=\frac{1}{9}\left(\frac{1}{\sqrt{15}}\right)^{8}\left(\frac{1}{5}\right)^{5 n-14} \\
H \chi\left(P_{n}\left[P_{2}\right]\right)=\frac{2}{9}+\frac{8}{15}+\frac{5 n-14}{25} .
\end{gathered}
$$

Theorem 12. Let $T_{n, k}$ be a Tadpole graph. Then

$$
\begin{gathered}
\chi\left(T_{n, k}, x\right)=(n+k-4) x^{\frac{1}{2}}+3 x^{\frac{1}{\sqrt{6}}}+x^{\frac{1}{\sqrt{2}}} \\
P \chi\left(T_{n, k}\right)=\left(\frac{1}{2}\right)^{n+k-4}\left(\frac{1}{\sqrt{6}}\right)^{3}\left(\frac{1}{\sqrt{2}}\right) \\
H \chi\left(T_{n, k}\right)=\frac{n+k-4}{4}+1 .
\end{gathered}
$$




\section{References}

[1] N. De, Sk.Md. Abu Nayeem, Computing the F-index of nanostar dendrimers, Pasific Science Review A: Natural Science and Engineering. 18(1) (2016) 14-21.

[2] B. Furtula, I. Gutman, A forgotten topological index, J. Math. Chem. 53(4)(2015) 1184-1190.

[3] M. Ghorbani, N.Azimi, Note on multiple zagreb indices, Iran J. Math. Chem. 3(2)(2012) 137-143.

[4] I. Gutman, K.C. Das, Some properties of the second Zagreb index, Match Common. Math. Comput. Chem. 52 (2004) 103-112.

[5] I. Gutman, N. Trinajstic, Graph theory and molecular orbitals. III. Total $\pi$-electron energy of alternant hydrocarbons, Chem. Phys. Lett. 17(4) (1972) 535-538.

[6] H. Lowell Hall, B. Lemont Kier, Molecular connectivity in chemistry and drug research, Boston: Academic Press, 1976. ISBN 0-12-406560-0

[7] M.K. Siddiqui, M. Imran, A. Ahmad, On zagreb indices, zagreb polynomials of some nanostar dendrimers, Appl. Math. comput. 280 (2016) 132-139.

[8] G.H. Shirdel, H.R. Pour, A.M. Sayadi, The hyper-zagreb index of graph operations, Iran J. Math. Chem. 4(2) (2013) 213-220.

[9] H.J. Wiener, Structural determination of paraffin boiling points, J. Amer. Chem. Soc. 69(1) (1947) $17-20$. 